\title{
MS39-05 | At the Intersection of Crystallography AND Spectroscopy - Studies of Selected Photoactive Coinage Metal Complexes
}

Jarzembska, Katarzyna (Department of Chemistry, University of Warsaw, Warsaw, POL); Kaminski, Radoslaw (Department of Chemistry, University of Warsaw, Warsaw, POL); Laski, Piotr (Department of Chemistry, University of Warsaw, Warsaw, POL); Szarejko, Dariusz (Department of Chemistry, University of Warsaw, Warsaw, POL); Drapala, Jakub (Department of Chemistry, Warsaw University of Technology, Warsaw, POL); Durka, Krzysztof (Department of Chemistry, Warsaw University of Technology, Warsaw, POL)

Polynuclear transition-metal complexes frequently constitute the active sites of both biological and chemical catalysts, provide access to unique chemical transformations derived from specific metal $\cdots$ metal interactions and cooperation. Among this group, the $\mathrm{d}^{8}$ or $\mathrm{d}^{10}$ transition-metal coordination compounds are worth mentioning due to their interesting optoelectronic properties. Nevertheless, conscious design of functional materials of specific and controllable properties, requires a deep understanding of the nature of physical phenomena behind them.

Hence, the current project was dedicated to investigations of light-induced processes in selected coinage metal complexes. Emphasis was put on the direct determination of structural changes via advanced experimental methods supplemented by theoretical computations. The studied systems include both the literature-reported complexes as well as newly designed and synthesised ones. All of them exhibit luminescent properties which were carefully examined. The determined Stokes shifts and emission decay times were used to plan the time-resolved photocrystallographic experiments. The laser-induced excited states were probed with both 100 ps and 250 ns delay times at the 14-ID-B BioCARS APS beamline using the pink-Laue X-ray radiation. The results of such investigations are presented.

Overall, the project constitutes continuation of our studies on silver-copper complexes, in which we attempted to deepen the nature of the excited state, relate structural and spectroscopic properties of the system and analyse the effects of high pressure.

The authors thank NSC (2014/15/D/ST4/02856) and WCSS (grant No. 285) in Poland, EU programme (POIG.02.01.00-14-122/09) and APS, USA (DOE: DE-AC02-06CH11357, NIH: R24GM111072) for financial support and access to facilities. 